\title{
Ambulant erworbene Pneumonie: Wer muss stationär behandelt werden?
}

\begin{abstract}
Eine wichtige und manchmal schwierige Entscheidung im Management von Patienten mit einer ambulant erworbenen Pneumonie (CAP) ist die Frage, ob eine stationäre Behandlung erforderlich ist. Um die Entscheidung zu erleichtern, wurden verschiedene Tools zur Risikostratifizierung von Patienten mit einer CAP entwickelt und validiert, z. B. CURB-65, Pneumonia Severity Index (PSI), SMART-COP u. a. Sie bieten eine objektive Hilfestellung bei der Beantwortung dieser wichtigen Frage.
\end{abstract}

— Eine pneumonieassoziierte Hypoxie ist ein häufiger Grund für eine stationäre Einweisung. Dennoch ist sie bislang nicht Bestandteil der gängigen Risikostratifizierung. Vor diesem Hintergrund haben Majumdar und Kollegen untersucht, welchen Einfluss eine Hypoxämie auf die Prognose von ambulant behandelten Patienten mit einer CAP hat.

In die prospektive Untersuchung haben die Autoren in den Jahren 2000 bis 2002 alle Patienten aus sieben Notfallstationen in Edmonton (Kanada) aufgenommen, bei denen eine CAP diagnostiziert wurde und die anschließend ambulant behandelt wurden. Als unabhängige Variable diente die pulsoxymetrisch

gemessene Sauerstoffsättigung $\left(\mathrm{SaO}_{2}\right)$. Der primäre Endpunkt war die 30-TageMortalität und/oder eine spätere Hospitalisierung.

Von 2923 Patienten lagen die vollständigen Daten vor. Alle Patienten hatten eine radiologisch bewiesene CAP, 96\% erhielten eine antibiotische $\mathrm{Be}$ Leitlinien. Die mittlere $\mathrm{SaO}_{2}$ betrug $95 \%$, wobei $2 \%$ der Patienten eine $\mathrm{SaO}_{2}$ $<88 \%$ aufwiesen, $4 \%$ eine $\mathrm{SaO} 2<90 \%$ und $11 \%$ eine $\mathrm{SaO}_{2}<92 \%$. Patienten mit einer $\mathrm{SaO}_{2}<92 \%$ waren älter, wiesen mehr Begleiterkrankungen auf und hatten einen höheren PSI-Wert.

Die Gesamtmortalität innerhalb der handlung entsprechend den aktuellen

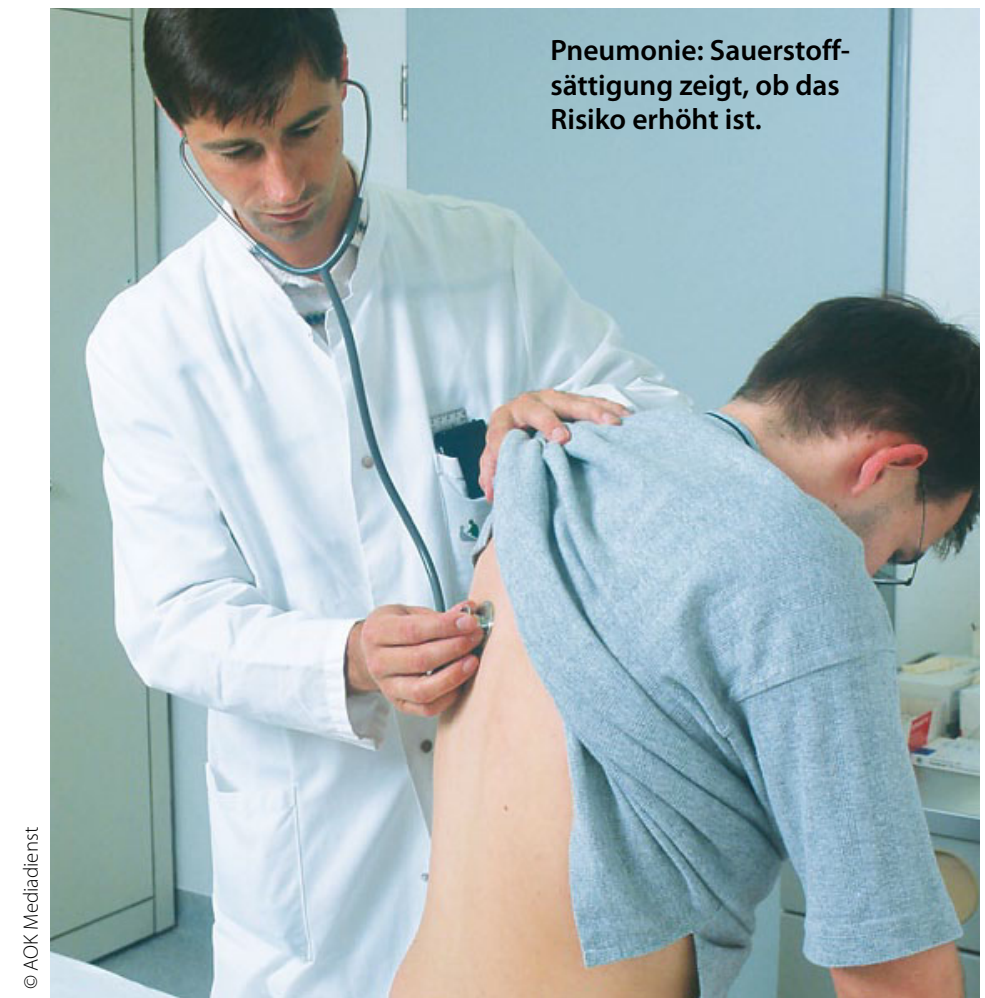

ersten 30 Tage betrug 1\%. 8\% der Patienten mussten nachträglich stationär aufgenommen werden. Patienten mit einer $\mathrm{SaO}_{2}<90 \%$ mussten im Verlauf signifikant häufiger stationär aufgenommen werden als Patienten mit einer höheren $\mathrm{SaO}_{2}$ (18 vs. 7\%; $\left.\mathrm{p}<0,001\right)$. AuBerdem hatten sie eine signifikant höhere Mortalitätsrate (6 vs. $1 \%$; p < 0,001). Im Hinblick auf den zusammengesetzten Endpunkt (Mortalität oder Hospitalisierung) war der Unterschied noch deutlicher (21 vs. $8 \%$ ).

\section{Kommentar}

Üblicherweise ist eine Hypoxämie eine Kontraindikation für die ambulante Behandlung von Patienten mit CAP. Leider existieren bislang nur wenige gute Daten, die das Risiko für eine schlechtere Prognose bei dieser Patientengruppe klar quantifizieren oder sichere Cut-off-Werte definieren. Somit liefert diese Studie einen wichtigen Beitrag, um die Versorgung von CAP-Patienten in der Zukunft sicherer zu gestalten.

Patienten mit einer $\mathrm{SaO}_{2}<90 \%$ haben demzufolge ein deutlich erhöhtes Risiko für einen schlechteren Verlauf ihrer Erkrankung. In der Multivariatanalyse erwies sich eine $\mathrm{SaO}_{2}<90 \%$ mit einer adjustierten OR von 1,7 als unabhängiger Risikofaktor für einen schlechteren Verlauf. Diese Assoziation war ab einer Sättigung $\geq 92 \%$ nicht mehr nachweisbar. Somit sollte die Messung der $\mathrm{SaO}_{2}$ fest als Ergänzung zu den Scoringsystemen in das Management von CAP-Patienten integriert werden.

Diese Ergebnisse rechtfertigen die stationäre Aufnahme von CAP-Patienten mit einer Sauerstoffsättigung $<92 \%$, auch wenn sie nach dem CURB-65 oder dem PSI einer niedrigen Risikogruppe angehören.

S. OTT =

\section{- S. R. Majumdar et al.}

Oxygen saturations less than $92 \%$ are associated with major adverse events in outpatients with pneumonia: a population-based cohort study. Clin Infect Dis. 52 (2011) 325-331 